\title{
Designing Short-Term Wave Traces to Assess Wave Power Devices
}

\author{
Schmitt, P., Danisch, L., Lamont-Kane, P., \& Elsaesser, B. (2018). Designing Short-Term Wave Traces to \\ Assess Wave Power Devices. International Journal of Offshore and Polar Engineering, 28(1), 46-53. \\ https://doi.org/10.17736/ijope.2018.as23
}

\section{Published in:}

International Journal of Offshore and Polar Engineering

\section{Document Version:}

Publisher's PDF, also known as Version of record

\section{Queen's University Belfast - Research Portal:}

Link to publication record in Queen's University Belfast Research Portal

Publisher rights

t @ by The International Society of Offshore and Polar Engineers

\section{General rights}

Copyright for the publications made accessible via the Queen's University Belfast Research Portal is retained by the author(s) and / or other copyright owners and it is a condition of accessing these publications that users recognise and abide by the legal requirements associated with these rights.

Take down policy

The Research Portal is Queen's institutional repository that provides access to Queen's research output. Every effort has been made to ensure that content in the Research Portal does not infringe any person's rights, or applicable UK laws. If you discover content in the Research Portal that you believe breaches copyright or violates any law, please contact openaccess@qub.ac.uk. 


\title{
Designing Short-Term Wave Traces to Assess Wave Power Devices
}

\author{
Pál Schmitt \\ Queen's University Belfast \\ Portaferry, UK \\ Lucas Danisch \\ TU Hamburg-Harburg \\ Hamburg, Germany
Paul Lamont-Kane and Björn Elsäßer
Queen's University Belfast \\ Portaferry, UK
}

\begin{abstract}
It is generally accepted that an estimate of mean power capture for a wave energy converter (WEC) in a given sea state can only be obtained over many hundreds (or thousands) of wave cycles. The difficulty stems from the fact that WECs typically exhibit significant nonlinearities in their responses. A reduction in the number of wave cycles needed to obtain accurate results would allow the use of numerical tools for design optimization tasks that are currently too computationally demanding. In this paper, experimental time traces are analyzed to provide reasonable estimates of relative variations in device performance using short-duration sea states. We examine the suitability of various metrics of surface elevation time traces by comparing corresponding WEC data of interest. The results show that carefully selected wave traces can be used to reliably assess variations in power output due to changes in hydrodynamic design or wave climate. It is also demonstrated how confidence levels increase with running time, so in the future simulations could be run until sufficient accuracy is achieved to choose the best design.
\end{abstract}

\section{INTRODUCTION}

One of the most common methods used in the development of wave energy converters (WECs) is physical experimentation undertaken in a wave tank, which can be both time consuming and expensive.

Oscillating wave surge converters (OWSCs) are designed to be deployed in the near shore region in water depths of approximately $12-15 \mathrm{~m}$ and utilize the amplified surge motion of water surface waves in this region to pitch back and forward about a hinge mounted on the seabed. The basic concept is shown in Fig. 1.

One design feature of OWSCs is the shape of the side edges (as shown in Fig. 2). The thickness of the edges of the flap affects energy loss, as a result of viscous effects, and thus power capture (Cameron et al., 2010).

The utilization of computational fluid dynamics (CFD) simulations has become general practice in many areas of marine engineering. Correctly used, CFD tools achieve results with the same accuracy as physical testing (Schmitt and Elsäßer, 2015; Palm et al., 2016; Kim et al., 2015; Chen et al., 2017). However, in an industrial setting, and in contrast to experimental testing, CFD tools are still not capable of obtaining results for long-duration wave traces at a comparable cost (Schmitt et al., 2012). To receive valid and statistically significant data, calibrated seas often have running times longer than $256 \mathrm{~s}$ at the 40th scale. It is evident

Received November 1, 2016; updated and further revised manuscript received by the editors July 10, 2017. The original version (prior to the final updated and revised manuscript) was presented at the Twenty-sixth International Ocean and Polar Engineering Conference (ISOPE-2016), Rhodes, Greece, June 26-July 1, 2016.

KEY WORDS: Sea state, wave trace, experimental tank testing, wave parameters, nonlinear effects, statistics.

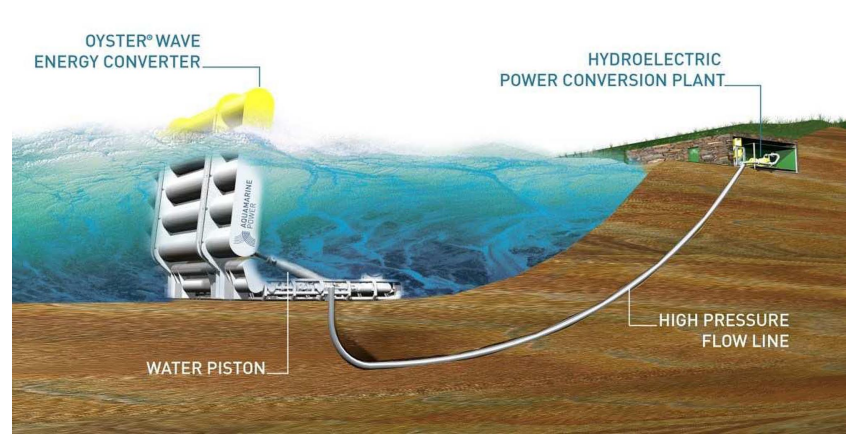

Fig. 1 Conceptual sketch of Oyster 1

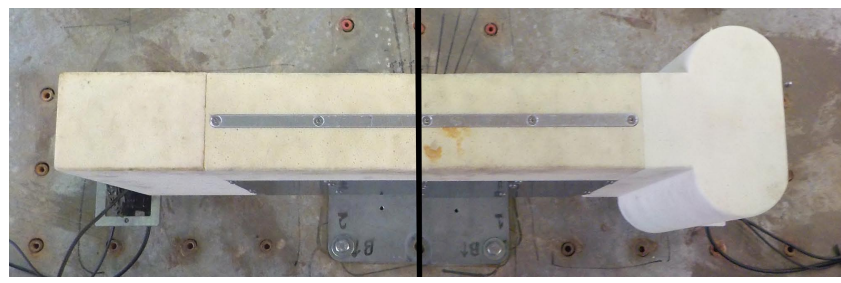

Fig. 2 40th scale model of common box-shaped flap shown without (left) and with (right) end-effectors

that simulations of that length would be infeasible in CFD in an industrial context.

Ransley et al. (2017) used CFD tools to investigate the extreme loads on a WEC. They concluded that, even for relatively shortterm events like wave impacts, the specific wave group combinations responsible for the most severe structural loads are unclear and can only be found by testing a range of conditions. 
Crooks et al. (2014), Asmuth et al. (2014), and Schmitt et al. (2016) demonstrated that, because of the strong nonlinear wave interaction of OWSCs, standard tools employing linearized potential methods cannot accurately predict behavior in realistic operating conditions.

Clabby et al. (2012) investigated the influence of spectral shapes on the power output of OWSCs in a series of tanktesting experiments. They found a decrease in power capture with increasing spectral bandwidth and also showed that power capture is most sensitive to spectral shape in shorter-period seas. The variation in power output was less than $25 \%$ that in the comparable monochromatic sea, while a variation of less than $6 \%$ between JONSWAP, Bretschneider, and bimodal spectra was found.

This paper investigates the relationship between the mean power output and properties of a surface elevation time trace, with the aim of finding the shortest possible time traces to be used for power output or power take-off (PTO) optimization of a WEC.

The investigation was performed in the following steps. First, a study was conducted on how specific metrics like significant wave height and mean period of a sea state converge with increasing length of the individual sea state. Then different metrics of surface elevation of a sea state were compared in order to identify a short excerpt within a full sea state that yields the same power output characteristics. Finally, the analysis is applied to data obtained from experimental wave tank tests for two different OWSC shapes and sea states. Particular emphasis is placed on the maximum power output deduced from power curves for different damping coefficients.

A surface-piercing OWSC was used in this investigation as an example; however, the methods should be equally applicable to the investigation of other structures deployed in the marine environment.

\section{GENERATION AND ANALYSIS OF A SEA STATE}

This section focuses on the data processing and wave-maker input definition. Details of tank geometry and further information are provided in the section EXPERIMENTAL SETUP, and the mathematical background is provided in the appendix. The clock rate $C R=32 \mathrm{~Hz}$ of the wave maker determines the rate of data output and data sampling in processes like wave generation and device triggering. To operate the tank, a run number $r n$ has to be chosen. The run number defines the time interval $T_{r}$ after which the generated surface elevation repeats.

$T_{r}=\frac{2^{r n}}{C R}$

The time vector $t$ with a sampling rate of $d t$ and a length of $T_{r}$ was defined to have the same number of data points $\kappa=1.3 \cdot 10^{5}$ for each run number.

$d t=\frac{T_{r}}{\kappa}$

Only frequency components $f_{n}$ with $d f$ as an even denominator can be implemented in a generated wave trace.

$f=n \cdot d f, \quad n \in \mathbb{N}$

The inverse of the repeat time gives the frequency interval $d f$ between components in the chosen frequency range that may be generated by the wave paddles (Rogers and Bolton King, 1997).

$d f=\left\{\begin{array}{l}1 / T_{r}, \\ f_{n+1}-f_{n}\end{array}\right.$

\begin{tabular}{lccc}
\hline & Sea04 & Sea10 & Sea19 \\
\hline$H_{m 0}[\mathrm{~m}]$ & $1.75\left(4.375 \cdot 10^{-2}\right)$ & $1.75\left(4.375 \cdot 10^{-2}\right)$ & $1.75\left(4.375 \cdot 10^{-2}\right)$ \\
$T_{m}[\mathrm{~s}]$ & $5.5(0.8696)$ & $7.5(1.185)$ & $9.5(1.502)$ \\
\hline
\end{tabular}

Table 1 Calibrated sea states. Values are given in full scale with model scale in parentheses.

The frequency range $f_{\min } \leqslant f_{n} \leqslant f_{\max }$ is restricted by the memory of the wave maker and was chosen according to the following conditions:

$$
\begin{gathered}
0.002 \geq \int_{0 H z}^{f_{\min }} S(f) d f \\
0.005 \geq \int_{f_{\max }}^{8 H z} S(f) d f
\end{gathered}
$$

Considering that spectral density is proportional to the energy contained in the respective frequency component, an energy loss of $0.7 \%$ is accepted by using this method. Because of the large tail of components with higher frequencies and low spectral densities, a greater part is cut off on the right side of the integral to achieve an adequate number of spectral components.

The final spectrum is then computed by inserting the received frequency range $f_{n}$ into Eq. 19 . From the new spectrum the amplitude components are extracted using Eq. 15. A random initial phase $\varphi_{n}$ for each frequency component must be chosen. The hereby defined parameters are then used in Eq. 14. The result is a unique surface elevation time trace.

The sea states displayed in Table 1 are going to be used for this investigation. They were chosen from 47 sea states used to describe the wave climate at the European Marine Energy Centre (EMEC) test site.

\section{CONVERGENCE STUDY OF SEA STATE PARAMETERS}

Since the phase shift $\varphi$ of spectral components is applied randomly, the statistical properties of numerically generated sea states vary. Therefore, a convergence study was conducted for the values of $\bar{H}, H_{1 / 3}$, and $T_{z m}$ for differing initial phase angles. One hundred sea states for nine different lengths, all defined by one JONSWAP spectrum computed by the user's input of $H_{m 0}$ and $T_{01}$, were analyzed to see how the standard deviation (std) of the time domain variables develops when the duration of the sea state increases. Power output, the property of interest in this investigation, is expected to be related to those values, but it should be noted that the exact nature of the dependence is unknown.

Results for Sea10 are shown in Figs. 3 and 4. The $x$-axis shows the lengths of the sea states going from $8 \mathrm{~s}$ to $2048 \mathrm{~s}$, doubling in each step. The left $y$-axis labels the magnitude of the mean values of the corresponding seas shown as circles and connected by a solid line. The standard deviations for each sea state are shown as error bars. The dash-dotted line shows the standard deviation $\sigma$ normalized to the respective mean value. It converges toward zero with increasing sea length. The dashed lines represent the input values of $H_{m 0}$ and $T_{01}$. Note that the values of $H_{1 / 3}$ and $T_{z m}$ are considerably lower. This is due to the usage of a spectrum that is not narrow-banded (Ochi, 1998).

The graphs help us to understand how accurately the input values are matched for a given length of a sea state. They also give an idea of the possible variance of the results of a single test 


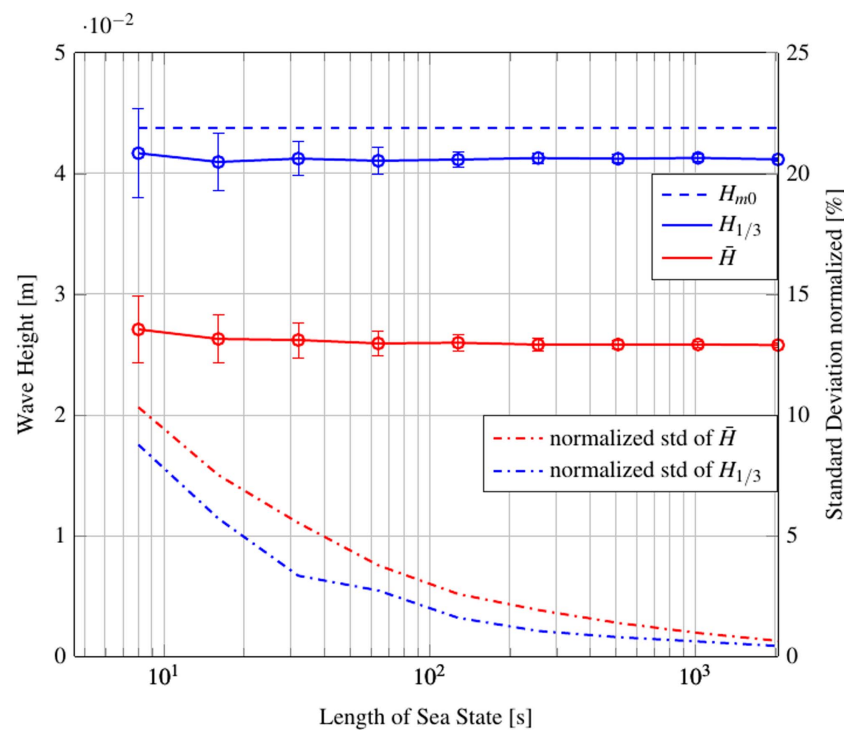

Fig. 3 Convergence of $H_{1 / 3}$ and $\bar{H}$ in generated sea states with length of seas. Error bars represent standard deviation.

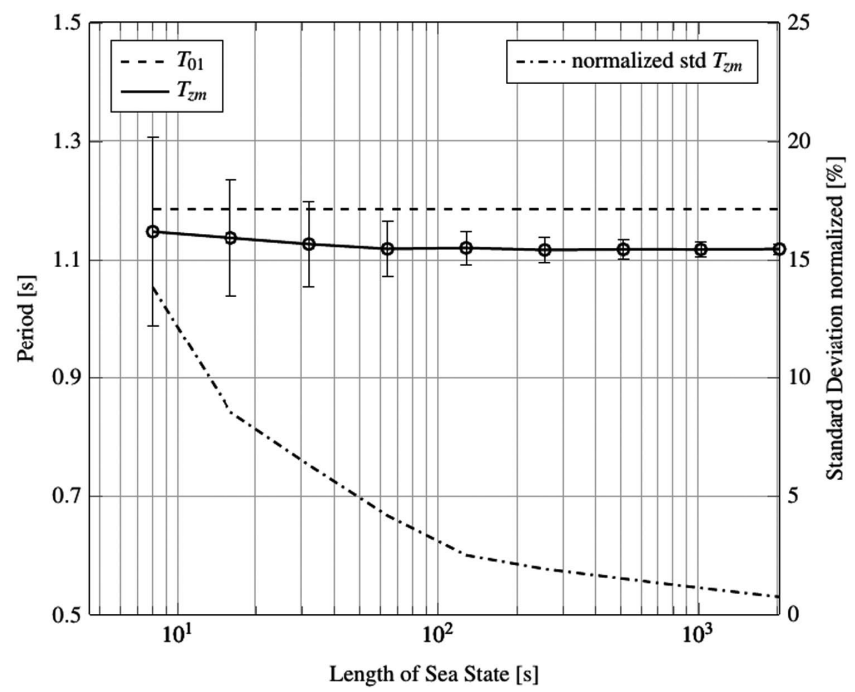

Fig. 4 Convergence of $T_{z}$ in generated sea states with length of seas. Error bars represent standard deviation.

run with the respective duration. The time-domain values of short duration sea states with lengths of less than $32 \mathrm{~s}$ have normalized standard deviations of more than $5 \%$. While sea states of more than $128 \mathrm{~s}$ already show standard deviations of less than $2.5 \%$, the values of the standard deviations of sea states longer than $2048 \mathrm{~s}$ are less than $1 \%$.

\section{ADVANCED METRICS}

Many metrics can be derived from the spectral variance of a given sea state or surface elevation. Whether spectral representation is appropriate or accurate enough at all is another interesting question and has been discussed recently by Saulnier et al. (2011).

Ohle et al. (2005) discussed the lack of information on statistics of wave height and period of single waves in spectral descriptions for the investigation of wave run-up. Since wave run-up is proportional to $T \sqrt{H}$, they recommended analyzing time traces for this combined parameter.
A Fourier analysis of a short-duration elevation trace often contains discrete peaks and is of limited use to derive spectral metrics. Time-domain metrics were thus used exclusively in this work. The investigation was performed by comparing metrics of a short length or so-called window of surface elevation to the same metric of the entire sea state. These wave traces were then assessed by comparing average power production during these short time traces to the value obtained over the long run.

It should be mentioned that, obviously, many trivial solutions exist. An infinite number of single wave cycles will create the same average power as a sea state. Our goal, however, is to find a metric over several wave cycles, such that the wave parameters in the trace have some causal relationship to the entire sea state and are of physical relevance. The following section describes metrics developed in addition to the well-known parameters discussed above to analyze the data gained from the experiments. All postprocessing was performed in Matlab/GNU Octave.

\section{Metric Comparison Method}

In wave energy research, significant wave height $H_{1 / 3}$ is often used for calculations. However, this metric describes the mean wave height of only the largest one-third of waves. Two-thirds of significant data are thus not properly taken into account. One can easily think of examples of wave traces with the same significant wave height but very different overall mean wave heights $\bar{H}$. Mean wave period $T_{z m}$ influences power output as well. Therefore, all three were included in a newly developed metric.

This method combines the values of the time-domain variables for each window and compares them to the full sea state's values. The significant wave height of the current window $H_{1 / 3}(w)$ is subtracted from the significant wave height of the whole sea state $H_{1 / 3 \text {, sea }}$ and likewise for the other two variables. The absolute value of the difference is then divided by the desired target value of the whole sea state to receive a proportion of the window's discrepancy. This normalization makes the single discrepancies comparable. They are summed up equally and form the discrepancy metric $\Delta_{m}$.

$$
\begin{aligned}
\Delta_{m}(w)= & \frac{\left|H_{1 / 3, \text { sea }}-H_{1 / 3}(w)\right|}{H_{1 / 3, \text { sea }}}+\frac{\left|\bar{H}_{\text {sea }}-\bar{H}(w)\right|}{\bar{H}_{\text {sea }}} \\
& +\frac{\left|T_{z m, \text { sea }}-T_{z m}(w)\right|}{T_{z m, \text { sea }}}
\end{aligned}
$$

However, two of the three summands are values of wave height and only one is a value of wave period. Therefore, a change of wave height will influence $\Delta_{m}$ more strongly. A simple weighted average would solve the raised issue. The window with the smallest $\Delta_{m}$ is assumed to be the most similar.

\section{Wave Height Histogram Comparison Method}

An alternative metric used is based on the distribution of wave heights. Each sea state has a certain number of waves with respective heights as well as wave lengths that can be displayed graphically in a histogram (Fig. 5). It is plotted as the fraction of occurrence (quantity allocated to a bin divided by the total quantity) over the corresponding wave height or period, for the study of power output focus lies on the histogram of wave heights. The bin size is defined for each sea state by dividing the highest wave by seven, which also specifies the number of bins. This value was chosen such that histograms of short-duration windows with fewer 


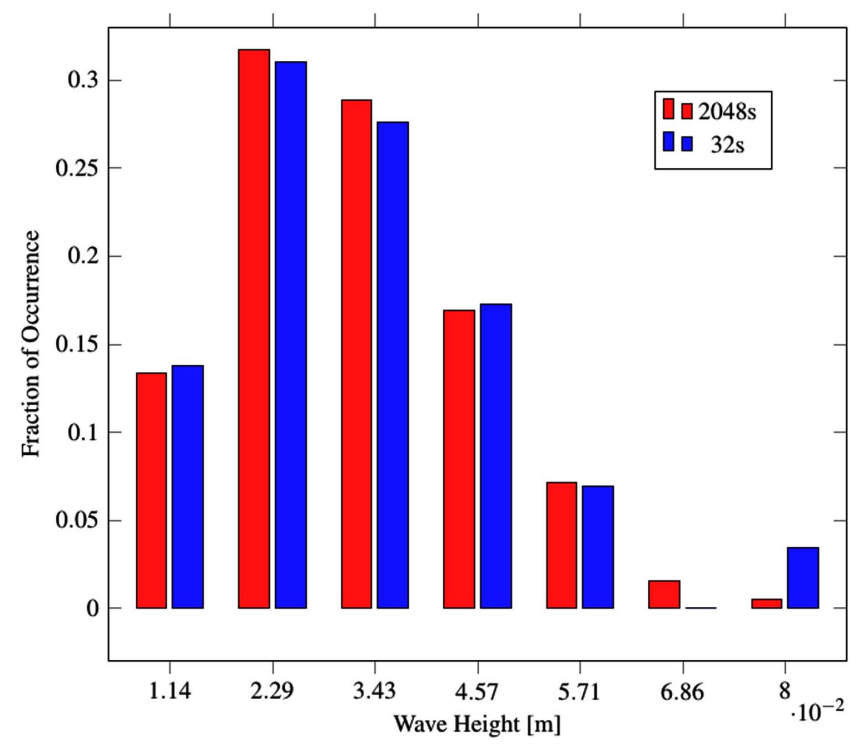

Fig. 5 Histogram of wave heights of a full sea state of $2048 \mathrm{~s}$ and similar extract of $32 \mathrm{~s}$ duration

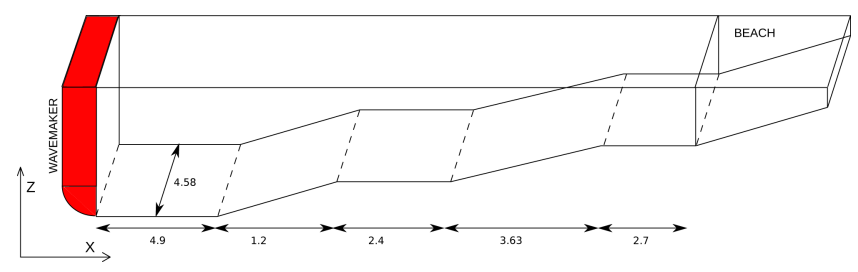

Fig. 6 Schematic drawing of the Queen's University Belfast (QUB) tank, dimensions in [m]

than 30 waves would still show a reasonable shape. A scalar metric $\Delta_{h}$ can then be computed as follows:

$\Delta_{h}(w)=\sum_{i=1}^{7}\left|\beta_{i}(w)-\beta_{\text {sea }, i}\right| \cdot \varepsilon_{i}$

where $\beta_{i}$ is the current bin's fraction of occurrence and $\beta_{\text {sea, } i}$ is the corresponding bin's fraction of the full sea state. The value of the variable $\epsilon_{i}$ is the bin's maximum wave height. They are multiplied to the corresponding absolute difference to add weight to discrepancies of bins with greater wave height, since they are believed to have higher influence on power output.

\section{EXPERIMENTAL SETUP}

To investigate the performance of the methods described in the previous sections, experimental tank tests were performed. The setup is very similar to the one used by Asmuth (2014), so only the most important details are given here. Tests were performed at the 40th scale, and Froude scaling applied.

The wave tank of the Marine Research Group is $4.58 \mathrm{~m}$ wide and $20 \mathrm{~m}$ long. The water depth is $0.691 \mathrm{~m}$ at the deepest point. Between the horizontal testing areas, the concrete tank floor gradually slopes up toward the beach. The 20th scale area is located at $6.1 \mathrm{~m}$ from the paddles and $0.15 \mathrm{~m}$ above the deepest point. The 40th scale area starts $12.1 \mathrm{~m}$ from the paddles and $0.35 \mathrm{~m}$ from floor level. In full scale, that resembles a water depth of $13.4 \mathrm{~m}$ for the 40th scale model (see Fig. 6). The tank's wave maker module consists of six wave paddles engineered by Edinburgh Design Ltd. Generated waves are mainly absorbed by the beach at the

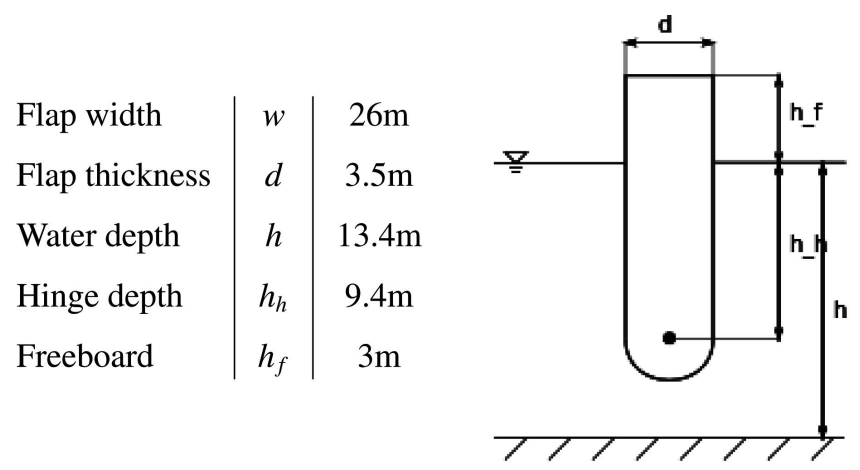

Fig. 7 Definition of geometric parameters in full scale

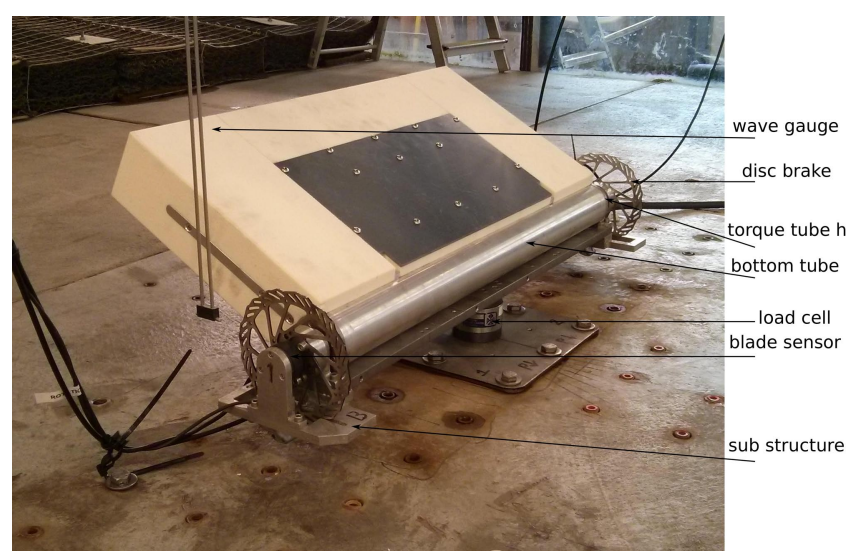

Fig. 8 Setup of model flap, substructure, damping mechanism, and measuring equipment in the wave tank

opposite end. The wave paddles detect and absorb reflected waves as well. Detailed information about the tank and beach setup can be found in Henry (2009) and Rogers and Bolton King (1997).

The 40th scale OWSC model designed by Asmuth (2014) was used for the experiments. Its geometry is shown in Figs. 2 and 7. The simple box-shaped flap (EE1) can easily be interchanged to a flap with end effectors (EE3) without draining the tank and recalibrating the wave gauges. Therefore, different performances of the two designs and the relationship of power output to the given wave trace can be recorded efficiently for each sea state.

The model consists of four foam blocks with aluminium frames for added stability. Furthermore, PVC plates compress the foam core. The assembled model is mounted to a bottom tube. Within it lie the torque transducers directly connected to the axis, which is held by bearings on the substructure.

Flap rotation, damping torque, and surface elevation have been measured at a sampling frequency of $128 \mathrm{~Hz}$. The full assembly of equipment is shown in Fig. 8. To evaluate the performance of a given OWSC, different loads are applied to the brakes, simulating the power take-off (PTO) system during testing. Power $P$ is obtained as the product of torque $\tau$ and rotational velocity $\omega$.

$P=\tau \cdot \omega$

The power curve $P\left(\tau_{\text {rms }}\right)$ is a polynomial fitted through points of the arithmetic mean of power $P$ over the root mean square (RMS) of instantaneous torque $\tau_{\text {rms }}$.

$\tau_{\mathrm{rms}}=\sqrt{\frac{1}{T_{r}} \int_{0}^{T_{r}}[\tau(t)]^{2} d t}$ 


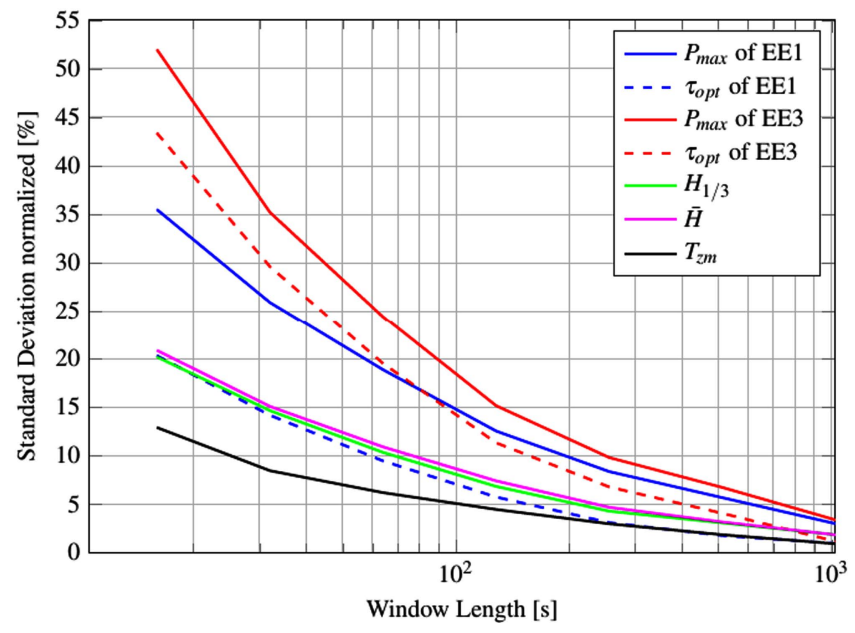

Fig. 9 Standard deviations for individual window lengths of torque, power output, and single time domain metrics

Tests were run for different flap shapes and sea states. For each configuration, the ideal damping level was found as described in detail in Schmitt et al. (2016). Added end effectors showed a significant increase of performance compared to the standard flap.

\section{RESULTS}

\section{Standard Deviations}

The following standard deviation plot presents the conventional metrics mean wave height $\bar{H}$, significant wave height $H_{1 / 3}$, and mean zero up-crossing period $T_{z m}$, as well as maximum power $P_{\max }$ and optimum RMS torque $\tau_{\text {opt }}$ (Fig. 9). All values are normalized to the mean of each data point. The standard deviations were deduced from the range of values of the numerous windows analysed. The figure shows the convergence of all metrics with increasing window lengths for both modifications of the model.

The individual time-domain variables are independent of the tested model, since they are derived from the elevation trace of the sea state. It is noticeable that the standard deviation of the mean zero up-crossing period shows similar values to those in the convergence study on fully spectral sea states. The standard deviations of mean and significant wave height of the windows are twice as high. For comparison, see Figs. 3 and 4.

Optimum RMS torque and maximum power output are influenced by the type of flap used for the experiments. Shape EE3 causes the values to have a higher standard deviation on the shortduration windows, especially for optimum torque.

\section{Evaluation of Method Accuracy}

The methods described allow us to sort the analyzed wave trace windows according to the lowest respective metric of discrepancy under the assumption that the best fit will show a power output very similar to the one obtained from the full $2048 \mathrm{~s}$ sea state. The methods have been applied to various window lengths, providing a succession of assessed excerpts for each. The "best" window's power output is plotted in Figs. 10 and 11 as circles connected by a solid line over the corresponding window length. The boxshaped model is shown in blue, and the end-effector model is shown in red. The dashed lines represent the average power output of the entire sea state $P_{\max \text {, sea }}$ as a reference (see Figs. 10 and 11).

It is somewhat challenging to assess the quality and the accuracy of each method in a statistical approach. If we look at the 10 closest windows (a small fraction of $0.5 \%-1.0 \%$ of the number of analyzed windows) under the assumption that the methods do find excerpts that will produce a more similar power output than a random window, then they should all yield similar results. Therefore, it is assumed that the closer the best window is to $P_{\text {max, sea }}$ and the smaller the standard deviation of the 10 closest windows is, the better the method is. A modified standard deviation $\sigma_{m}$ was developed to express the average distance of the best window's $P_{\max , i}$ to the value of the full sea $P_{\max , \text { sea }}$.

$\sigma_{m}=\sqrt{\frac{1}{n-1} \sum_{i=1}^{n}\left(P_{\max , i}-P_{\max , \mathrm{sea}}\right)^{2}}$

$\sigma_{m}$ is shown as error bars for each point and subsequently normalized to $P_{\max \text {, sea }}$ as displayed with the dash-dotted line.

We observe for both methods that the agreement of the points with the dashed line gets better with growing window length. Also, the standard deviation $\sigma_{m}$ converges toward zero. Keeping in mind that the standard deviation of $P_{\max }$ of all windows behaves likewise, this might not be surprising; however, if we compare Figs. 10 and 11 to Fig. 9, we see that the methods' standard deviation values of power converge faster. This suggests that they are not scattering much around the desired value. The standard deviation of the metric comparison method does not decline as consistently. A peak is observable at $128 \mathrm{~s}$ window length. Furthermore, it is striking that the curves correlate. Whenever the best window of a certain length overestimates the power output for EE1, it is also overestimated for EE3 by that window in a similar magnitude. The offsets $\theta$ are shown in Tables 2 and 3 .

$\theta=1-\frac{P_{\max , i}}{P_{\max , \text { sea }}}$

The Pearson correlation coefficient between the offsets of EE1 and EE3 to their respective $P_{\text {max, sea }}$ was also evaluated for the firstbest to the 10th-best window of each method to show the continuity of this observation (Fig. 12). Values close to one describe a strong correlation. Figure 12 shows that for both methods the correlation factor is never lower than 0.94 . The offsets of the histogram comparison method have a better correlation in general.

To evaluate which of the two presented methods yields more accurate results, the arithmetic mean of the absolute offset

$\Theta=\frac{1}{n} \sum_{i=1}^{n}\left|1-\frac{P_{\max , i}}{P_{\max , \mathrm{sea}}}\right|$

of the 10 best windows for each window length has been evaluated for each method, respectively. As a reference, the same has been done for all windows. The result is shown in Fig. 13. It can be observed that the mean absolute offsets of both methods are similar and significantly lower than the mean absolute offset of a randomly chosen window. The curve of the wave height histogram comparison method declines more steadily than the one of the metric comparison method. The $16 \mathrm{~s}$ windows in general have a $\Theta$ of approximately $30 \%$ while the 10 best by each method have a $\Theta$ of approximately $10 \%$. Windows that are $128 \mathrm{~s}$ long have a $\Theta$ of approximately $12 \%$ while the 10 best windows of the histogram comparison method have a $\Theta$ of approximately $4 \%$. The arithmetic mean of the absolute offset converges toward zero more rapidly for the excerpts identified by the methods developed in this work than it does for the excerpts as a whole. The methods identify excerpts that are on average $30 \%$ closer to the reference power output than a randomly chosen excerpt. Shortduration wave traces of $32 \mathrm{~s}$ achieve $\Theta$ values of less than $10 \%$. 


\begin{tabular}{lcccccccc}
\hline & \multicolumn{6}{c}{ Window Length [s] } \\
\cline { 3 - 9 } & 16 & 32 & 64 & 128 & 256 & 512 & 1024 \\
\hline \multirow{2}{*}{ Offset $\theta[\%]$} & EE1 & 6.64 & 7.30 & -2.16 & 9.18 & 2.31 & -0.11 & -0.83 \\
& EE3 & 7.84 & 12.37 & -3.28 & 8.32 & 1.72 & 0.038 & -0.90 \\
\hline
\end{tabular}

Table 2 Offset from maximum power of full sea for best windows of metric comparison method

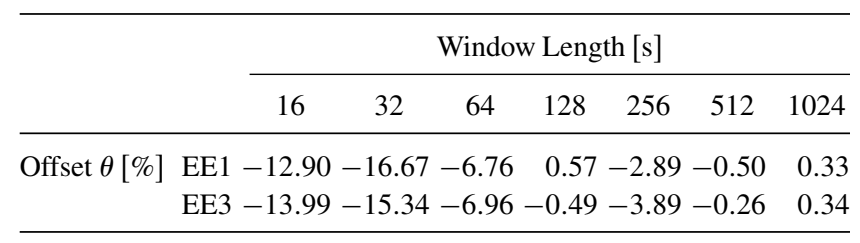

Table 3 Offset from maximum power of full sea for best windows of histogram comparison method

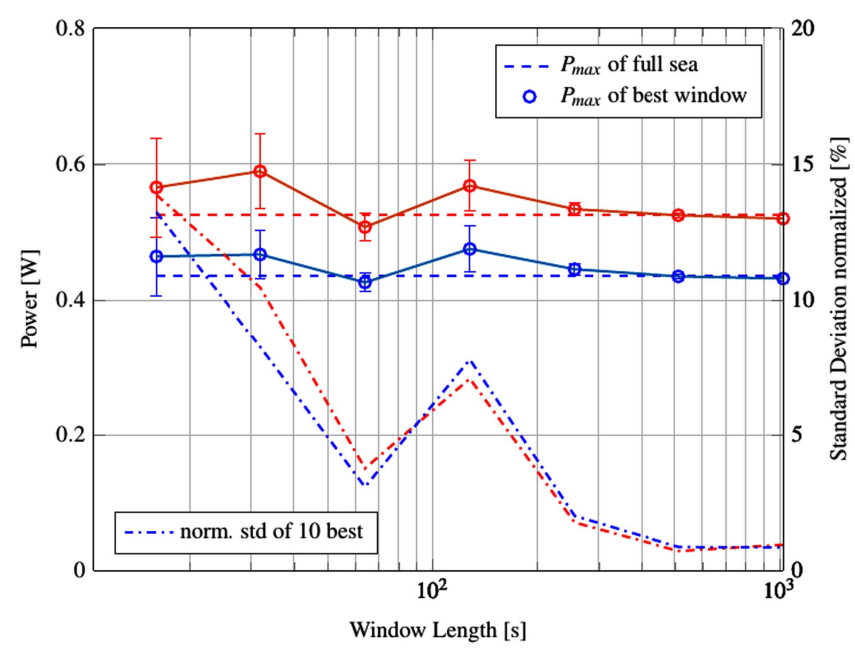

Fig. 10 Power output of best window found with metric comparison method over window length. Standard deviation of 10 best windows shown as error bars, blue EE1 and red EE3.

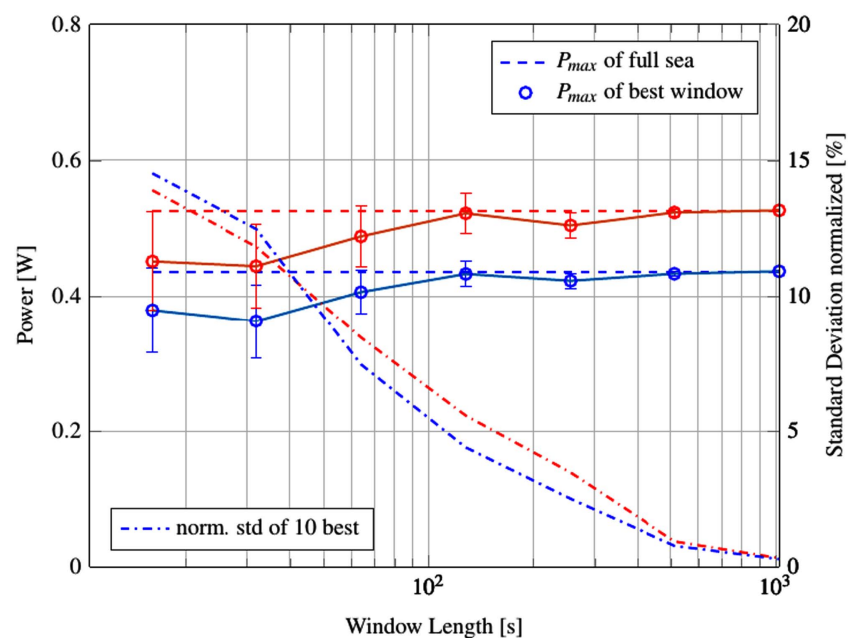

Fig. 11 Power output for the best window found with histogram method over window length. Standard deviation of 10 best windows shown as error-bars, blue EE1 and red EE3.

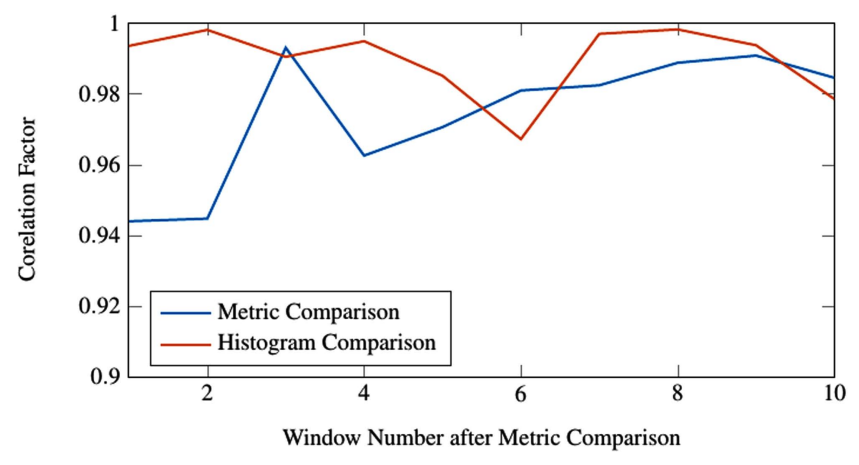

Fig. 12 Correlation between EE1 and EE2 curve of optimum power from Figs. 10 and 11 for 10 best windows

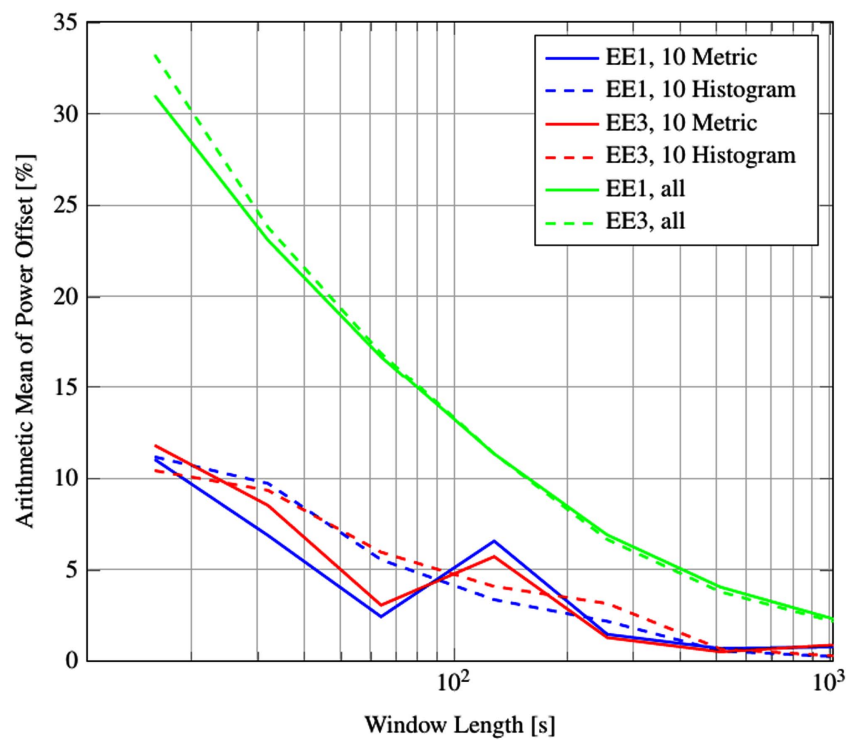

Fig. 13 Arithmetic mean of absolute power offset $\Theta$ of 10 best windows of each method and of all windows as a reference line

\section{CONCLUSIONS}

This paper presents an investigation into the assessment of power output of different OWSC designs using short-duration wave traces. Short-duration windows can be used to evaluate relative improvement of performance for design changes on model shapes with significant differences.

Excerpts exist within a full sea state that match the maximum power output within a few percents. Whether they do so by pure chance or by distinctive properties should be reviewed in more detail.

While not discussed in detail here, under the assumption of linear superposition, statistical properties like the distribution of wave heights can be derived analytically (Longuet-Higgins, 1957) and have been found to be fairly accurate when compared to offshore measurements (Petrova and Soares, 2009; Nayak and Panchang, 2015). It should be noted, however, that the vast majority of those validation studies is mostly concerned about the highest occurring waves, while energy production estimates of WECs are rather based on the average wave height.

The methods can evaluate short-duration excerpts of the full sea state and filter out windows that lead to a significantly better estimation of the maximum power output than a random window. The wave height histogram comparison method generally shows 
a more consistent decline of its mean absolute offset of the 10 closest windows to the histogram of the full sea state. The methods developed in this work are capable of providing wave traces for CFD that achieve a power output value with an accuracy of approximately $10 \%$ for windows of $32 \mathrm{~s}$ or longer and $5 \%$ for windows of $128 \mathrm{~s}$ or longer for the given sea states.

As the research on metrics describing single waves or wave groups is developing continuously, more metrics become available for testing. A metric's suitability is also likely to depend on the mode of operation of the WEC.

Additional experiments are required to investigate the exact dependency of the bandwidth and shape of the spectrum on the accuracy of the proposed method. If one considers monochromatic seas as extremely narrow banded spectra, for which a single wave would give exact results, it seems intuitively right to assume that the more frequency bands need to be evaluated the more combinations are possible. Clabby et al. (2012) also attributed the decreased power output in wide bandwidth spectra to the difficulty in finding good damping levels for each incoming wave cycle.

A greater variety of model shapes may be included with shapes of greater and finer difference to make an assumption on the information value delivered by the power production assessment with the developed methods. For the given OWSC main dimensions and wave spectra, a CFD evaluation of designs using shortduration wave traces of manageable length could now be conducted. The required simulation time can be adjusted dynamically until the difference between two designs is within the levels of confidence.

\section{ACKNOWLEDGEMENTS}

Lucas Danisch's work was supported by the Mobility Fund of TU Hamburg-Harburg. Their support is much appreciated.

\section{REFERENCES}

Asmuth, H (2014). The Influence of End Effector Shape on the Dynamics of the Oscillating Wave Surge Converter Oyster, Master's Thesis, University of Technology Hamburg, Hamburg, Germany.

Asmuth, H, Schmitt, P, Henry, A, and Elsäßer, B (2014). “Determination of Non-Linear Damping Coefficients of BottomHinged Oscillating Wave Surge Converters Using Numerical Free Decay Tests," in: Renewable Energies Offshore, Guedes Soares, C (Ed), Taylor \& Francis Group, 507-514.

Cameron, L, et al. (2010). "Design of the Next Generation of the Oyster Wave Energy Converter," Proc 3rd Int Conf Ocean Energy, Bilbao, Spain, ICOE, 12 pp.

Chen, W, et al. (2017). "Numerical Modeling of a PointAbsorbing Wave Energy Converter in Irregular and Extreme Waves," Appl Ocean Res, 63, 90-105. https://doi.org/10.1016/j.apor.2017.01.004.

Clabby, D, Henry, A, Folley, M, and Whittaker, T (2012). "The Effect of the Spectral Distribution of Wave Energy on the Performance of a Bottom-Hinged Flap-Type Wave Energy Converter," Proc 31st Int Conf Ocean Offshore Arct Eng, Rio de Janeiro, Brazil, ASME, 7, 331-339. https://doi.org/10.1115/OMAE2012-83398.

Crooks, D, Whittaker, TJ, van't Hoff, J, and Cummins, C (2014). "Experimental Validation of Numerically Generated Wave Excitation Torque on an OWSC," in: Renewable Energies Offshore, Guedes Soares, C (Ed), Taylor \& Francis Group, 523-530.
Goda, Y (2010). Random Seas and Design of Maritime Structures, World Scientific, $732 \mathrm{pp}$.

Hasselmann, K, et al. (1973). Measurements of Wind-Wave Growth and Swell Decay During the Joint North Sea Wave Project (JONSWAP), Report, Deutsches Hydrographisches Institut, Hamburg, Germany, 12, 93 pp.

Henry, A (2009). The Hydrodynamics of Small Seabed-Mounted Bottom-Hinged Wave Energy Converters in Shallow Water, $\mathrm{PhD}$ Thesis, Queen's University Belfast, UK.

Kim, B-H, Wata, J, Zullah, MA, Ahmed, MR, and Lee, Y-H (2015). "Numerical and Experimental Studies on the PTO System of a Novel Floating Wave Energy Converter," Renewable Energy 79, 111-121.

https://doi.org/10.1016/j.renene.2014.11.029.

Longuet-Higgins, MS (1957). "The Statistical Analysis of a Random, Moving Surface," Philos Trans $R$ Soc London Ser A, 249(966), 321-387. https://doi.org/10.1098/rsta.1957.0002.

Nayak, S, and Panchang, V (2015). "A Note on Short-Term Wave Height Statistics, Aquat Procedia, 4, 274-280.

https://doi.org/10.1016/j.aqpro.2015.02.037.

Ochi, MK (1998). Ocean Waves, Cambridge University Press, Cambridge, UK.

https://doi.org/10.1017/CBO9780511529559.

Ohle, N, Daemrich, K-F, and Tautenhain, E (2005). "Influence of Spectral Shape on Wave Parameters and Design Methods in Time Domain," Ocean Waves Measurement and Analysis, 5th Int Symp WAVES, Madrid, Spain, 150.

Palm, J, Eskilsson, C, Paredes, GM, and Bergdahl, L (2016). "Coupled Mooring Analysis for Floating Wave Energy Converters Using CFD: Formulation and Validation,' Int J Mar Energy, 16, 83-99. https://doi.org/10.1016/j.ijome.2016.05.003.

Petrova, PG, and Guedes Soares, C (2009). "Probability Distributions of Wave Heights in Bimodal Seas in an Offshore Basin," Appl Ocean Res 31(2), 90-100. https://doi.org/10.1016/j.apor.2009.06.005.

Ransley, E, Greaves, D, Raby, A, Simmonds, D, and Hann, M (2017). "Survivability of Wave Energy Converters Using CFD," Renewable Energy 109, 235-247.

https://doi.org/10.1016/j.renene.2017.03.003.

Rogers, D, and Bolton King, G (1997). Wave Generation Using Ocean and Wave, Manual version 3.62, Edinburgh Designs Ltd. www.edesign.co.uk.

Saulnier, J-B, et al. (2011). "Wave Groupiness and Spectral Bandwidth as Relevant Parameters for the Performance Assessment of Wave Energy Converters," Ocean Eng 38(1), 130-147. https://doi.org/10.1016/j.oceaneng.2010.10.002.

Schmitt, P, and Elsäßer, B (2015). "On the Use of OpenFOAM to Model Oscillating Wave Surge Converters," Ocean Eng, 108, 98-104. https://doi.org/10.1016/j.oceaneng.2015.07.055.

Schmitt, P, Asmuth, H, and Elsäßer, B (2016). "Optimising Power Take-Off of an Oscillating Wave Surge Converter Using High-Fidelity Numerical Simulations," Int J Mar Energy, 16, 196-208. https://doi.org/10.1016/j.ijome.2016.07.006.

Schmitt, P, Doherty, K, Clabby, D, and Whittaker, T (2012). "The Opportunities and Limitations of Using CFD in the Development of Wave Energy Converters," Mar Offshore Renewable Energy Conf, London, UK, 89-97. 


\section{APPENDIX: MATHEMATICAL DESCRIPTION OF A SEA STATE}

Sea waves form a unique surface elevation $\eta$ that can, according to linear theory, be described by a superposition of an infinite number of sinusoidal waves. Mathematically, the surface elevation $\eta$ of the ocean can therefore be defined as the sum of an infinite number of sinusoids:

$\eta=\sum_{n=1}^{k} A_{n} \sin \left(-2 \pi f_{n} t+\varphi_{n}\right)$

with individual amplitudes $A_{n}$, frequencies $f_{n}$, and random phase shifts $\varphi_{n}$. The time vector is given by $t$. The spectral density $S_{n}$ for each amplitude component $A_{n}$ derives from the following equation:

$S_{n}=\frac{A_{n}^{2}}{2 d f}$

Several metrics are made available by analyzing a given time trace of a surface elevation. Metrics of this kind will be referred to as analytical or statistical metrics.

- The wave height of a sine curve is the double of the respective amplitude. Wave height $H$ in an irregular time trace is the elevation difference between the trough and the crest while crossing zero once, going from positive to negative values (zero upcrossing). The arithmetic mean of all wave heights in a time span will be referred to as $\bar{H}$.

- A metric often used to describe a sea condition is the average wave height of the largest third of all waves $H_{1 / 3}$, also referred to as "significant wave height."

- Mean wave period $T_{z m}$ is defined as the arithmetic mean of the time intervals between zero up-crossing incidents in the surface elevation.

Applying a Fourier analysis to a given time trace of a surface elevation yields a spectral representation of the sea state. Generally it is displayed as spectral density $S(f)$ over frequency $f$. Metrics derived from the spectral representation will be referred to as spectral metrics. Particularly useful is the spectral moment of the density function. $m_{i}$ describes the $i$ th moment of the frequency spectrum and is defined as

$m_{i}=\int_{0}^{\infty} f^{i} S(f) d f$
The significant wave height can then be derived from the spectral moment:

$H_{m 0}=4 \sqrt{m_{0}}$

The factor four in front of the square root in Eq. 17 is only valid for narrow-banded frequency spectra. This relationship yields to overestimations of approximately $1.5 \%-8 \%$ for more broadbanded frequency spectra (Ochi, 1998).

The mean period is calculated by the division of the zeroth moment and the first moment:

$T_{01}=\frac{m_{0}}{m_{1}}$

Several formulations have been proposed to describe frequency spectra established through analysis from accumulated field data. However, spectral characteristics vary depending on the geographical location where the data were recorded. A modification of the JONSWAP spectrum, which is based on the results of the Joint North Sea Wave Observation Project (Hasselmann et al., 1973, as presented in Goda, 2010), is given here:

$S(f)=\beta_{J} H_{m 0}^{2} T_{p}^{-4} f^{-5} \exp \left[-\frac{5}{4}\left(T_{p} f\right)^{-4}\right] \gamma^{\exp \left[-\left(T_{p} f-1\right)^{2} /\left(2 \sigma^{2}\right)\right]}$

with

$\beta_{J}=\frac{0.0624}{0.230+0.0336 \gamma-\frac{0.185}{1.9+\gamma}}[1.094-0.01915 \cdot \ln \gamma]$

$T_{p} \simeq \frac{T_{01}}{1-0.532(\gamma+2.5)-0.569}$

$\sigma=\left\{\begin{array}{l}0.07: f \leq f_{p}, \\ 0.09: f \geq f_{p}\end{array}\right.$

$\beta$ is shown here as used in the procedure for wave tank calibration. It is a relationship among fetch length, mean wind speed, and gravity. The peak wave period $T_{p}$ is estimated from $T_{01}$ by Eq. 21. The peak enhancement factor $\gamma$ is set to one for all results shown. 